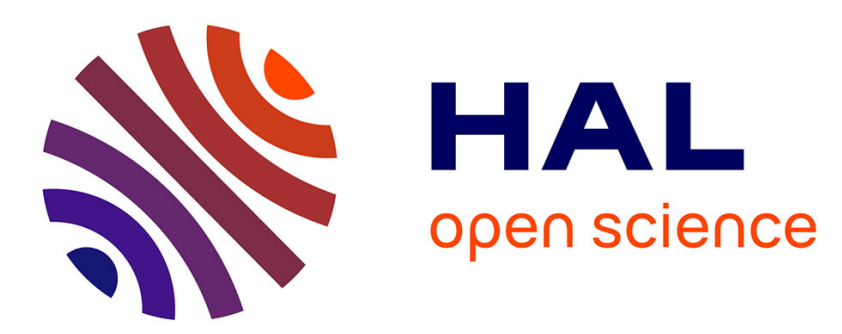

\title{
DARS2 mutations in mitochondrial leukoencephalopathy and multiple sclerosis
}

P Isohanni, T Linnankivi, J Buzkova, T Lönnqvist, H Pihko, L Valanne, P J

Tienari, I Elovaara, T Pirttilä, M Reunanen, et al.

\section{- To cite this version:}

P Isohanni, T Linnankivi, J Buzkova, T Lönnqvist, H Pihko, et al.. DARS2 mutations in mitochondrial leukoencephalopathy and multiple sclerosis. Journal of Medical Genetics, 2010, 47 (1), pp.66. 10.1136/jmg.2009.068221 . hal-00552703

\section{HAL Id: hal-00552703 https://hal.science/hal-00552703}

Submitted on 6 Jan 2011

HAL is a multi-disciplinary open access archive for the deposit and dissemination of scientific research documents, whether they are published or not. The documents may come from teaching and research institutions in France or abroad, or from public or private research centers.
L'archive ouverte pluridisciplinaire HAL, est destinée au dépôt et à la diffusion de documents scientifiques de niveau recherche, publiés ou non, émanant des établissements d'enseignement et de recherche français ou étrangers, des laboratoires publics ou privés. 


\section{DARS2 mutations}

\section{in mitochondrial leukoencephalopathy and multiple sclerosis}

P Isohanni, ${ }^{1,2}$ T Linnankivi, ${ }^{2} \mathrm{~J}$ Buzkova, ${ }^{1}$ T Lönnqvist, ${ }^{2}$ H Pihko, ${ }^{2}$ L Valanne, ${ }^{3} \mathrm{PJ}$

Tienari, ${ }^{1,4}$ I Elovaara, ${ }^{5}$ T Pirttilä, ${ }^{6}$ M Reunanen, ${ }^{7}$ K Koivisto, ${ }^{8}$ S Marjavaara, ${ }^{1}$ A

Suomalainen $^{1,4}$

${ }^{1}$ Research Programme of Molecular Neurology, Biomedicum-Helsinki, University of Helsinki, Helsinki, Finland

${ }^{2}$ Department of Child Neurology, Helsinki University Central Hospital, Helsinki,

Finland

${ }^{3}$ Helsinki Medical Imaging Center, University of Helsinki, Helsinki, Finland

${ }^{4}$ Department of Neurology, Helsinki University Central Hospital, Helsinki, Finland

${ }^{5}$ Department of Neurology, University of Tampere and Tampere University Hospital,

Tampere, Finland

${ }^{6}$ Department of Neurology and Neuroscience, University of Kuopio and Kuopio

University Hospital, Kuopio, Finland

${ }^{7}$ Department Neurology, University of Oulu and Oulu University Hospital, Oulu,

Finland

${ }^{8}$ Central Hospital of Seinäjoki, Seinäjoki, Finland

Number of characters in title: 74

Number of words in abstract: 248

Number of words in the body of manuscript: 2683

Number of figures: 2

Number of tables: 1

Supplemental data: Supplementary table 1 
Correspondence to: Dr P Isohanni, Department of Child Neurology, Hospital for Children and Adolescents, Helsinki University Central Hospital, P.O.Box 280

(Lastenlinnantie 2), FIN-00029 HUS, Finland; e-mail pirjo.isohanni(at)helsinki.fi, tel. +358505447778

Disclosure: The authors report no conflicts of interest.

Key words: leukoencephalopathy, ataxia, axonal neuropathy, mitochondrial disease, multiple sclerosis 
Background: Leukoencephalopathy with brain stem and spinal cord involvement and high brain lactate (LBSL) was first defined by characteristic MRI and spectroscopic findings. The clinical features include childhood or juvenile-onset slowly progressive ataxia, spasticity, and dorsal column dysfunction, occasionally accompanied by learning difficulties. Mutations in DARS2, encoding mitochondrial aspartyl-tRNA synthetase, were recently shown to cause LBSL. The signs and symptoms show some overlap with the most common leukoencephalopathy of young adults, multiple sclerosis (MS).

Objective: To clarify the molecular background of LBSL patients in Finland, and to look for DARS2 mutations in a group of MS patients.

Methods: Clinical evaluation of LBSL patients, DARS2 sequencing and haplotype analysis, and carrier frequency determination in Finland.

Results: All eight LBSL patients were compound heterozygotes for DARS2 mutations: all carried R76SfsX5 change, seven had M134_K165del and one had C152F change. Axonal neuropathy was found in five of the eight patients. The carrier frequencies of the R76SfsX5 and M134_K165del mutations were 1:95 and 1:380, respectively. All patients shared common European haplotypes, suggestive of common European LBSL ancestors. No enrichment of the two common DARS2 mutations was found in $321 \mathrm{MS}$ patients.

Conclusion: All LBSL patients were compound heterozygotes, which suggests that $D A R S 2$ mutation homozygosity may be lethal or manifest as a different phenotype. We show here that despite identical mutations the clinical picture was quite variable in the patients. Axonal neuropathy was an important feature of LBSL. DARS2 mutations cause childhood-to-adolescence-onset leukoencephalopathy, but they do not seem to be associated with MS. 


\section{INTRODUCTION}

"Leukoencephalopathy with brain stem and spinal cord involvement and high brain lactate" (LBSL) is a recently described white matter disease with distinct findings in magnetic resonance imaging (MRI) and spectroscopy [1,2]. The disease manifests during childhood or young adulthood $[3,4]$, progresses slowly and affects the pyramidal tract, cerebellum and spinal cord. Brain MRI findings are unique hemispheric patchy or confluent white matter changes with involvement of specific brain stem and spinal cord tracts - and, in the majority of cases, proton magnetic resonance spectroscopy $\left({ }^{1} \mathrm{H}-\mathrm{MRS}\right)$ shows elevated lactate in abnormal brain white matter, whereas serum and cerebrospinal fluid lactate and other laboratory investigations are normal. LBSL was shown to be caused by mutations in DARS2, a mitochondrial aspartyl transfer-RNA synthetase [5]. Scheper et al reported 38 patients from 30 families, with compound heterozygous mutations in the DARS2 gene.

We studied the molecular background of Finnish patients with typical LBSL-findings in MRI and spectroscopy. Furthermore, we studied the role of DARS2 mutations in multiple sclerosis (MS), which is the most common disease affecting the white matter in young adults, and previously suggested as a differential diagnosis for LBSL [6]. 


\section{PATIENTS AND METHODS}

\section{Patients and samples}

We studied DNA samples of eight patients, four males and four females, with the clinical diagnosis of LBSL, based on typical MRI findings (supplementary table 1). Six patients were of Finnish, one of Finnish-Estonian and another of Russian origin, and all the parents were non-consanguineous. All patients were investigated by us at Helsinki University Central Hospital, and all samples were taken for diagnostic purposes. Informed consent for DNA analysis was obtained from the patients or their parents, and the study was approved by the Ethics Committee for Paediatrics, Adolescent Medicine and Psychiatry of Helsinki University Central Hospital. We also studied 321 MS trio families; 784 DNA samples including the patient and one or both parents. The patients were diagnosed as "clinically or laboratory supported definite MS" according to Poser criteria, and included both relapsing-remitting and progressive MS cases. The patients were collected in the University Central Hospitals of Helsinki $(n=113)$, Tampere $(n=89)$, Kuopio $(n=17)$, and Oulu $(n=60)$, as well as in Seinäjoki Central Hospital $(n=42)$ in Finland. The mean year of birth of the patients was 1959 (range 1934-1983), female:male ratio 2.4 and the percentage of HLADR15-positive patients 57\%. The samples were obtained after informed written consent. The genetic analysis of the MS patients was approved by the Ethics Review Board for Ophthalmology, Otorhinolaryngology, Neurology and Neurosurgery in the Hospital District of Helsinki and Uusimaa.

\section{LBSL DNA analysis}

The human chromosome 1 reference assembly sequence, which contains DARS2 genomic sequence (NT_004487) and DARS2 mRNA (NM_018122) were used as 
reference sequences. Genomic DNA was extracted from peripheral blood samples by using standard procedures. The coding sequence and the intron-exon boundaries of exons 3, 5 and 17 of DARS2 were amplified by polymerase chain reaction (PCR) using previously described primers[5]. For DNA sequence analysis we used the BigDye Terminator Ready Reaction Kit v.3.1 on an ABI3730XL DNA Analyzer (Applied Biosystems). Sequence processing was done with Sequencher 4.5 software (GeneCodes).

\section{MS and control DNA analysis}

To determine the carrier frequency of the mutations found in the patients from Finnish origin, we screened 375 anonymous DNA samples from the Finnish control individuals and 784 samples from MS families. We analyzed the presence of the p.R76SfsX5 (c.228-20_21delTTinsC) and p.M134_K165del (c.492+2T>C) mutations by PCR and solid-phase minisequencing as described [7]. The primer sequences for c. 228-20_21delTTinsC were 5'-TTGCATGGATTTTTGTTTGC-3' and 5'-BioTTGAACAAGCCCATCGAAAT- 3', and the primer sequences for c.492+2T>C were 5'-bio- TTCTGAATGCCTGCAAGAAG-3', and 5'TTCATGATGTGTCTACAATAAAATGC-3'. The detection primer for c.228-

20_21delTTinsC was

5'-GAGATTTTATCTTAAAATGTTTCTT-3' and for c.492+2T>C 5'-TATTAATAACAGAGGTTGGT-3'.

The complete DARS2 coding sequence and the intron-exon boundaries of those MS patients, who carried either of the two LBSL-associated mutations as heterozygous, were determined. The primer sequences were as in [5].

\section{Haplotype analysis}


Isohanni P et al. 7

We analyzed five polymorphic dinucleotide/trinucleotide markers flanking the DARS2 gene (D1S2790, D1S1589, D1S218, D1S2691 and D1S416). We added TAATACGACTCACTATAGGGAGA-tag to the 5'tail of forward primer of every marker (tag-forward-primer), as well as the same tag alone with FAM-label (FAM-tagprimer), which was used to label the PCR-product during the amplification. Fragment analysis was performed in ABI3730XL DNA Analyzer (Applied Biosystems) with GeneScan LIZ-500 size standard, and the data was analyzed with GeneMapper 4.0 software (Applied Biosystems). 


\section{RESULTS}

\section{Clinical manifestations}

Table 1 summarizes the clinical features and variation in disease progression. Data of patients 1-5 were partially reported in the paper by Linnankivi et al. [2] As a previously un-emphasized feature we noted that five of the eight patients exhibited signs of peripheral axonal neuropathy (Table 1). The overall clinical picture was surprisingly heterogeneous, considering the homogeneous genetic background of the patients. Therefore, we describe here the detailed clinical findings in two patients (P3 and P7), with quite different clinical presentations. 
Isohanni $\mathrm{P}$ et al. $\quad 9$

Table 1. Clinical characteristics of our LBSL patients

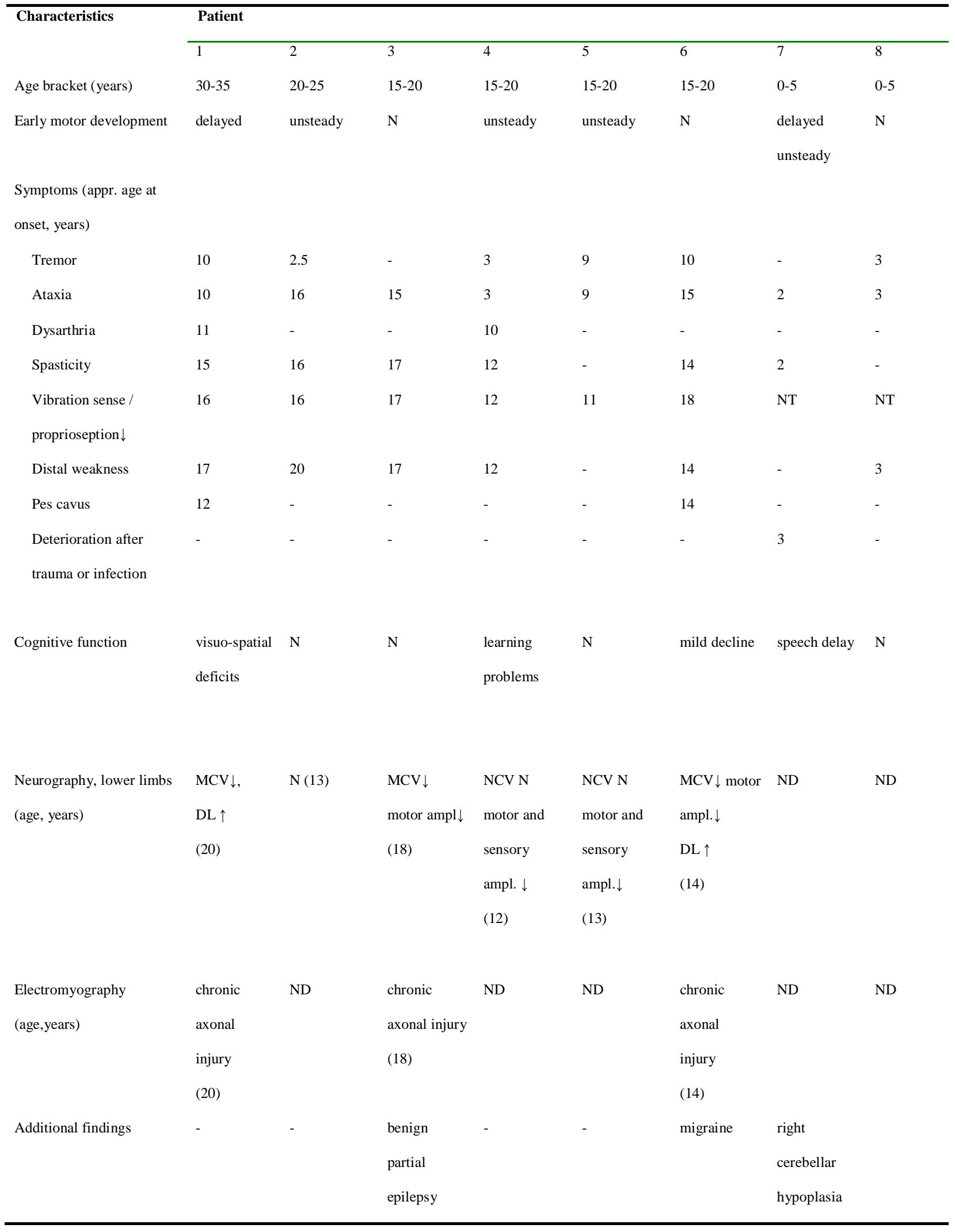


Abbreviations: $\mathrm{N}=$ normal, $\mathrm{nt}=$ not testable, $\mathrm{ND}=$ not done, $\mathrm{MCV}=$ motor conduction velocities, $\mathrm{NCV}=$ nerve conduction velocities, $\mathrm{DL}=$ distal latencies, ampl, $=$ amplitudes. 


\section{$\underline{\text { Patient } 3}$}

This 18-year-old boy had healthy parents and an affected elder brother (patient 2).

Pregnancy and birth of the patient were normal. He learned to walk at the age of 10 months and always had good motor skills. At the age of 8 years he had a few nocturnal epileptic seizures and an intensive left midtemporal spike focus in electroencephalography (EEG). Neurologic examination was normal, but MRI revealed abnormalities suggestive of LBSL (not shown). His seizures responded to oxcarbazepine, and medication was discontinued a few years later. Annual follow-up visits continued because of the MRI changes.

His cognitive skills and neurologic examination remained normal until the age of 15 years, when minimal ataxia and positive Babinski sign were recorded. At that time he was active in sports and his only complaint was pain and tremor in the legs after prolonged exercise. Distal spasticity and weakness of ankle dorsiflexion have developed only lately. Neurography was normal at the age of 13 years, but five years later it showed reduced motor conduction velocities with decreased motor amplitudes in lower limbs. Myography was consistent with axonal neuropathy, showing spontaneous activity in distal leg muscles, reduction in the number of motor units and large neuropathic units. MRI at the age of 18 years (Figure 1 A-E) showed mild progression of cerebellar and brain stem lesions.

\section{$\underline{\text { Patient } 7}$}

This 5-year-old boy was born at term to healthy parents. His elder brother is healthy. At the age of 19 months he was examined because of delayed motor development. He was ataxic and hypotonic. He learned to walk unsupported at the age of 2 years, but 
fell frequently. Distal spasticity, bilateral Babinski sign and hyperreflexia were

evident from the age of 2 years 3 months.

Brain MRI (figure 1 F-J) showed wide-spread white matter abnormalities typical for

LBSL in the supratentorial region, with minimal cerebellar and brain stem

involvement. Partial hypoplasia of the right cerebellar hemisphere was noted.

At the age of 3.5 years he had a minor trauma after falling from a chair. He did not lose consciousness, but was very tired, floppy and unable to walk for 4 days. Brain MRI, performed 2 weeks after the initial trauma, was unchanged. His walking gradually improved but did not return to the previous level and at present he is able to walk short distances independently. His fine motor skills are appropriate for age, but his speech development is mildly delayed.

\section{Variation in MRI findings}

Patients 4, 7 and 8, who had the earliest onset of symptoms, showed the most widespread brain MRI changes. Patient 7 with severe hemispheric changes had only subtle hyperintensity within the cerebellar white matter and lesions in the pyramids, and in addition to typical LBSL findings he had partial hypoplasia of the right cerebellar hemisphere (figure 1 F-J). MRS showed high choline with reduced NAA and a high lactate peak. In patient 3, MRI-findings preceded motor symptoms for several years. At the age of 8 years MRI showed abnormalities typical for LBSL [2], but minor ataxia appeared only 7 years later. MRI at age 18 showed some progression of the posterior fossa lesions, but no further change of the supratentorial white matter (figure 1 A-E). MRS showed no lactate and only a minor increase of choline was noted, although a lactate peak had been detected earlier.

\section{DNA analysis of LBSL patients}


All eight patients from 7 families had compound heterozygous mutations in DARS2.

All patients had a mutation in the 3'-region of intron 2, a TT deletion and C insertion (c.228-20_21delTTinsC). All patients had a second mutation in exon 5: the seven patients of Finnish origin had a $\mathrm{C}$ to $\mathrm{T}$ transversion at the splice site of exon 5 $(\mathrm{c} .492+2 \mathrm{~T}>\mathrm{C})$, and the patient of Russian origin had a $\mathrm{G}$ to $\mathrm{T}$ transversion at nucleotide 455 (c.455G>T).

\section{Carrier frequency}

We analyzed Finnish control chromosomes for the presence of the identified DARS2 mutations, and 6 chromosomes out of 560 carried the 228-20_21delTTinsC mutation, and 2 chromosomes out of 750 carried the c. $492+2 \mathrm{~T}>\mathrm{C}$ mutation. We estimated the carrier frequencies to be 1:95 for the c.228-20_21delTTinsC [p.R76SfsX5] mutation and 1:380 for the c.492+2T>C [p. M134_K165del] mutation in Finland.

\section{Haplotype analysis}

Figure 2 shows the results of the haplotype analysis. The patients shared a common core haplotype of three or four polymorphic markers, extending over a chromosomal region of $\sim 1,4 \mathrm{Mb}$. However, in some patients the shared haplotype extended to 2,1 Mb. These haplotypes were previously reported in patients from Europe and North America [5].

\section{DNA analysis of MS patients}

LBSL shows some overlapping clinical and neuroradiological features with MS. As the carrier frequency for the common DARS2 mutation c.228-20_21delTTinsC (p.R76SfsX5) was found to be considerably high (1:95), we tested the frequency of this and the M134_K165del variant in MS patient population. Four heterozygotes were identified in 321 MS families. The c.228-20_21delTTinsC mutation was found in two 
Isohanni P et al. 14

families, but only once transmitted from the parent to the MS patient. The c.492+2T>C (p.M134_K165del) mutation was found in two MS patients and in one parent (the sample from the putative carrier parent was not available for study). The frequencies of the two DARS2 mutations were 1:642 and 1:321 in MS families. These figures are lower than those in the general population (1:95 and 1:380). The three MS patients, heterozygous for a DARS2 mutation, did not carry other putative mutations in the complete DARS 2 coding region or in the exon-intron boundaries. The clinical picture of all these three MS patients was consistent with typical relapsing-remitting MS with the diagnosis at the ages of 24, 27 and 29. These results suggest that DARS2 mutations are not a significant risk factor of MS, and that LBSL was not misdiagnosed as MS in Finland. 


\section{DISCUSSION}

We report here eight LBSL patients, who were identified by typical MRI findings and who all had mutations in DARS2. Our report indicates that LBSL is a recognizable leukoencephalopathy that can be identified by typical MRI findings. Further, we show that all our LBSL patients were compound heterozygotes for DARS2 mutations, similar to a previous report [5]. This is unusual on the basis of the Finnish carrier frequencies (1:95 and 1:380), and suggests either that homozygosity for DARS2 mutations is embryonic lethal or manifests as a different, yet unidentified phenotype. We also show considerable variation in clinical symptoms, even in patients with identical mutations, as also seen in some other mitochondrial disorders [8]. This suggests that secondary predisposing / protecting genetic factors may affect the tissue responses to mitochondrial dysfunction and the disease manifestations.

The age of onset of LBSL varied from two to 15 years. Most patients learned to walk at normal age, but their gait remained unsteady, while the performance of some patients improved from initial manifestation. By adolescence, a mixture of upper and lower motor neuron, cerebellar, and dorsal column signs ensued. Most of the reported LBSL patients have become wheelchair-dependent in their teens or twenties [9], but all of our patients, six already young adults, are still able to walk. It is important to consider LBSL upon in patients with progressive white-matter disease of adolescence.

The white matter changes of LBSL are unique. The specificity of typical MRI pattern is emphasized by the fact that all our patients, whom we had identified by their MRI findings, also carried DARS2 mutations. This kind of symmetrical white matter lesions are unusual for mitochondrial disorders, where focal necrotic or stroke-like lesions, symmetrical deep gray matter lesions and cerebral or cerebellar atrophy are typical 
Isohanni $\mathrm{P}$ et al. 16

[10]. Axonal neuropathy - in contrast to demyelinating neuropathy found in conjunction with classical leukoencephalopathies - is recognised as an important feature of many mitochondrial disorders [11]. Axonal neuropathy was a common finding in the present series of LBSL patients with DARS2 mutations (Table 1). This feature was not initially described as part of the syndrome [1], but has been reported previously in four patients $[12,13]$. Axonal neuropathy in LBSL patients is comparable to that of NARP (neuropathy, ataxia and retinitis pigmentosa) and diseases caused by PEO1, POLG1 and MNF2 mutations, suggesting shared mechanisms underlying mitochondrial axonopathies. LBSL should be considered even in adult patients with slowly progressive ataxia and neuropathy.

Our patients were compound heterozygotes for three different DARS2 mutations. All had a intron-2 mutation, the most frequent mutation reported previously [5], accompanied by a splice site mutation in exon 5. The Finnish patients shared minimally a 2.1 Mb core haplotype with each other, indicating common ancestry. Perhaps surprisingly, however, as Finland is a genetic isolate, our patients shared a 1-2 Mb haplotype also with previously published Western European, Russian and North American DARS2-chromosomes, which indicated the existence of common, most likely European, ancestors. Similar pan-European ancestry has been found in other mitochondrial neurodegenerative disorders, such as mitochondrial recessive ataxia syndrome (MIRAS) $[8,14]$ and Friedreich's ataxia $[15,16]$. Similar to DARS2 mutations, also MIRAS-associated mtDNA polymerase gamma (POLG1) mutations show high carrier frequency in the population - 1:95 and 1:380 for DARS2 and 1:125 for MIRAS W748S mutation in Finland. Ancient haplotypes suggest that these housekeeping genes are not mutational hot-spots, and high carrier frequency suggests that carriership is not disadvantageous for getting progeny. 
Multiple sclerosis is the most common disorder affecting the white matter of young adults, and it has been mentioned as a differential diagnosis for LBSL [6]. Due to the relatively high carrier frequency of DARS2 mutations, and the lack of homozygotes, we studied patients with MS and their parents to test whether DARS2 mutations would be MS risk factors or whether some LBSL patients were phenocopies of MS. However, we found a considerably lower number of heterozygote DARS2 carriers in the MS population than in the control population. We conclude that the common DARS2 mutations are not common risk factors for MS.

LBSL, caused by mutations in a gene encoding mitochondrial aspartyl-tRNA synthetase, was the first reported human disease caused by a defect in a nuclearencoded protein affecting mitochondrial tRNA function. Recently Edvardson et al. reported mutations in a gene encoding mitochondrial arginyl-tRNA synthetase $(R A R S 2)$ in pontocerebellar hypoplasia with multiple mitochondrial respiratory chain defects [17]. One of our patients also had cerebellar hypoplasia, but this unilateral finding in a single patient may be coincidental. Both DARS2 and RARS2 mutations affect primarily the CNS, which has been suggested to be due to a tissue-specific vulnerability of the splicing machinery [17].

LBSL is a new disease among mitochondrial ataxias, and in Finland mutations in DARS2 are among the most common causes of childhood-onset leukoencephalopathy. So far most of the LBSL cases are diagnosed by paediatric neurologists, but since first symptoms can arise in adolescence or young adulthood, it is important also for adult neurologists to recognize this disease. Due to considerably high carrier frequency and the ancient common European origin of these haplotypes, the mutations are likely to be quite common also in other European-derived populations. 
FOOTNOTES

\section{Acknowledgements}

We thank the patients families for their cooperation, as well as Lilja Jansson for technical help, Janna Saarela for the control samples for carrier frequency determination, Pekka Ellonen for his skilful assistance with the analysis of haplotypes, and the following organizations for financial support: Academy of Finland (for AS, PJT and SKM), Helsinki University (for AS), Sigrid Juselius Foundation (for AS, PJT and HP), Foundation for Paediatric Research and Helsinki University Central Hospital EVO grant (for HP, TLi, PJT and PI).

\section{Competing interests}

None.

The Corresponding Author has the right to grant on behalf of all authors and does grant on behalf of all authors, an exclusive licence (or non exclusive for government employees) on a worldwide basis to the BMJ Publishing Group Ltd to permit this article (if accepted) to be published in Journal of Medical Genetics and any other BMJPGL products and sublicences such use and exploit all subsidiary rights as set out in the licence (http://jmg.bmjjournals.com/ifora/licence.pdf). 


\section{REFERENCES}

[1] van der Knaap MS, van der Voorn P, Barkhof F, Van Coster R, Krageloh-Mann I, Feigenbaum A, et al. A new leukoencephalopathy with brainstem and spinal cord involvement and high lactate. Ann Neurol 2003;53(2):252-258.

[2] Linnankivi T, Lundbom N, Autti T, Hakkinen AM, Koillinen H, Kuusi T, et al. Five new cases of a recently described leukoencephalopathy with high brain lactate. Neurology 2004;63(4):688-692.

[3] Petzold GC, Bohner G, Klingebiel R, Amberger N, van der Knaap MS, Zschenderlein R. Adult onset leucoencephalopathy with brain stem and spinal cord involvement and normal lactate. J Neurol Neurosurg Psychiatry 2006;77(7):889-891. [4] Labauge P, Roullet E, Boespflug-Tanguy O, Nicoli F, Le Fur Y, Cozzone PJ, et al. Familial, adult onset form of leukoencephalopathy with brain stem and spinal cord involvement: inconstant high brain lactate and very slow disease progression. Eur Neurol 2007;58(1):59-61.

[5] Scheper GC, van der Klok T, van Andel RJ, van Berkel CG, Sissler M, Smet J, et al. Mitochondrial aspartyl-tRNA synthetase deficiency causes leukoencephalopathy with brain stem and spinal cord involvement and lactate elevation. Nat Genet 2007;39(4):534-539.

[6] Hahn JS, Pohl D, Rensel M, Rao S, International Pediatric MS Study Group. Differential diagnosis and evaluation in pediatric multiple sclerosis. Neurology 2007;68(Suppl 2):S13-22.

[7] Suomalainen A, Syvanen AC. Quantitative analysis of human DNA sequences by PCR and solid-phase minisequencing. Mol Biotechnol 2000;15(2):123-131. 
[8] Hakonen AH, Heiskanen S, Juvonen V, Lappalainen I, Luoma PT, Rantamaki

M, et al. Mitochondrial DNA polymerase W748S mutation: a common cause of autosomal recessive ataxia with ancient European origin. Am J Hum Genet 2005;77(3):430-441.

[9] Schiffmann R, van der Knaap MS. The latest on leukodystrophies. Curr Opin Neurol 2004;17(2):187-192.

[10] Saneto RP, Friedman SD, Shaw DW. Neuroimaging of mitochondrial disease. Mitochondrion 2008;8(5-6):396-413.

[11] DiMauro S, Bonilla E, De Vivo DC. Does the patient have a mitochondrial encephalomyopathy? J Child Neurol 1999;14(Suppl 1):S23-35.

[12] Tavora DG, Nakayama M, Gama RL, Alvim TC, Portugal D, Comerlato EA. Leukoencephalopathy with brainstem and spinal cord involvement and high brain lactate: report of three Brazilian patients. Arq Neuropsiquiatr 2007;65(2B):506-511. [13] Uluc K, Baskan O, Yildirim KA, Ozsahin S, Koseoglu M, Isak B, et al. Leukoencephalopathy with brain stem and spinal cord involvement and high lactate: A genetically proven case with distinct MRI findings. J Neurol Sci 2008;273(1-2):118122.

[14] Hakonen AH, Davidzon G, Salemi R, Bindoff LA, Van Goethem G, Dimauro S, et al. Abundance of the POLG disease mutations in Europe, Australia, New Zealand, and the United States explained by single ancient European founders. Eur J Hum Genet 2007;15(7):779-783.

[15] Cossee M, Schmitt M, Campuzano V, Reutenauer L, Moutou C, Mandel JL, et al. Evolution of the Friedreich's ataxia trinucleotide repeat expansion: founder effect and premutations. Proc Natl Acad Sci U S A 1997;94(14):7452-7457.

[16] Montermini L, Andermann E, Labuda M, Richter A, Pandolfo M, Cavalcanti F, et al. The Friedreich ataxia GAA triplet repeat: premutation and normal alleles. Hum Mol Genet 1997;6(8):1261-1266. 
Isohanni $\mathrm{P}$ et al. 21

[17] Edvardson S, Shaag A, Kolesnikova O, Gomori JM, Tarassov I, Einbinder T, et

al. Deleterious mutation in the mitochondrial arginyl-transfer RNA synthetase gene is associated with pontocerebellar hypoplasia. Am J Hum Genet 2007;81(4):857-862. 


\section{LEGENDS TO FIGURES}

Figure 1. Variation of changes in T2-weighted MRI images in two different patients with the same DARS2 mutations. Fig. 1 A-E: Patient 3 at 18 years of age. The white matter lesions are patchy, located mainly along the trigones and frontal horns of the lateral ventricles. In the posterior fossa, characteristic lesions are seen along the intraparenchymal part of the trigeminal nerve $(\mathrm{C}$, short arrow), in the white matter around the dentate nuclei $(\mathrm{C}$, long arrow), inferior cerebellar peduncles and pyramids (D,arrows). The dorsal columns of the spinal cord are uniformly affected (E, arrow). Fig. 1 F-J: Patient 7 at 2.5 years of age. Extensive white matter involvement is seen in the centrum semiovale, sparing only the subcortical fibers. Abnormal signal is seen in the internal capsules. No lesions are seen in the posterior fossa, except for hypoplasia of the right cerebellar hemisphere, probably an unrelated phenomenon. The dorsal columns of the spinal cord are affected (J, arrow).

Figure 2. DARS2 haplotypes on chromosome 1 of the Finnish LBSL patients. Common haplotypes with the European patients [5] are indicated by light gray and dark gray boxes. Haplotype indicated by light gray box is the same as in patients from USA and Italy, and haplotype indicated by dark gray box is the same as in patients from Canada, Russia, Turkey and Eastern Europe [5]. 


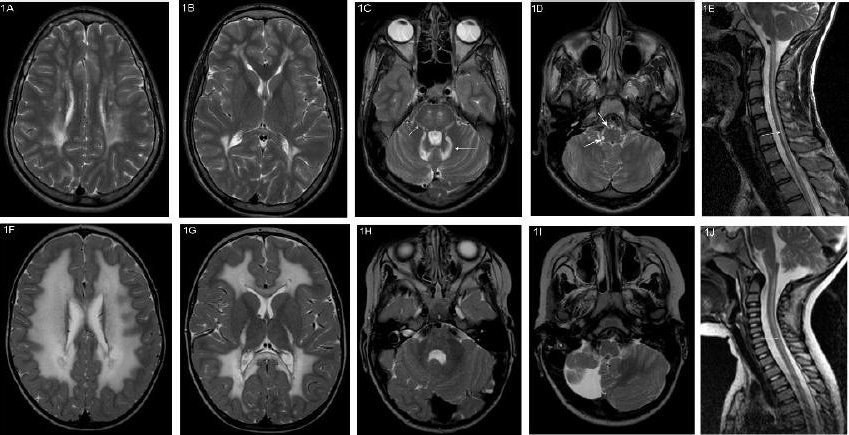




\begin{tabular}{|c|c|c|c|c|c|c|c|c|c|c|c|c|c|c|c|}
\hline \multirow{3}{*}{$\begin{array}{c}\text { DNA } \\
\text { marker } \\
\text { D152790 }\end{array}$} & \multirow{3}{*}{\begin{tabular}{|c|} 
Distance \\
from DARS2 \\
$800 \mathrm{~kb}$ \\
\end{tabular}} & \multicolumn{14}{|c|}{ R76Sf8X5/M134 K185del } \\
\hline & & \multicolumn{2}{|c|}{ P1 } & \multicolumn{2}{|c|}{$\mathrm{P}_{2}$} & \multicolumn{2}{|c|}{ P3 } & \multicolumn{2}{|c|}{ P4 } & \multicolumn{2}{|c|}{ P5 } & \multicolumn{2}{|c|}{ P6 } & \multicolumn{2}{|c|}{ P7 } \\
\hline & & 270 & 270 & 270 & 270 & 256 & 272 & 256 & 270 & 266 & 270 & 256 & 271 & 268 & 272 \\
\hline \multicolumn{16}{|c|}{ DARS2 } \\
\hline D15 & 30 & 223 & 24 & 223 & 24 & 223 & 241 & 223 & 24 & 223 & 241 & 223 & 24 & 223 & 223 \\
\hline D15218 & $b$ & $2 \pi 7$ & 269 & 277 & 269 & 277 & 269 & 277 & $28 \mathrm{~s}$ & 279 & 269 & 277 & $2 a s$ & 277 & $2 \pi 7$ \\
\hline D1S2691 & Mb & 275 & 287 & 275 & 287 & 275 & 285 & 275 & 285 & 275 & 285 & 275 & 285 & 275 & 275 \\
\hline 015416 & $1,3 \mathrm{Nb}$ & $1 \pi$ & 181 & 177 & 181 & 177 & 179 & 177 & 181 & 177 & 179 & 177 & 170 & 177 & 181 \\
\hline
\end{tabular}

